\title{
Tubo de PVC liso com diferentes envoltórios como material alternativo na drenagem subterrânea ${ }^{1}$
}

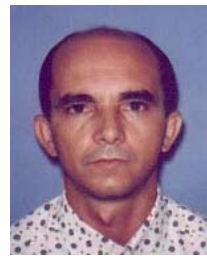

Florício P. de Almeida², Vera L. A. de Lima³, Carlos A. V. de Azevedo³, Karina de S. Andrade ${ }^{4} \&$ José Dantas Neto ${ }^{3}$

\footnotetext{
1 Parte da Dissertação de Mestrado do primeiro autor, apresentada à UFPB, desenvolvida com o apoio do CNPq

2 UEBA. E-mail: floricio@deag.ufpb.br (Foto)

${ }^{3}$ UFCG/CCT/DEAg. Av. Aprígio Veloso 882, Bodocongó, CP 10.087, CEP 58109-970, Campina Grande, PB

${ }^{4}$ Mestranda em Engenharia Agrícola, UFCG/CCT/DEAg. Bolsista da CAPES
}

Protocolo $165-20 / 11 / 2001$

\begin{abstract}
Resumo: Em regiões áridas e semi-áridas irrigadas a drenagem é, freqüentemente, necessária para prevenir o encharcamento do solo e o controle da salinidade. Um dos principais obstáculos na instalação de um sistema de drenagem subterrânea é o alto custo do investimento inicial. Assim, alguma contribuição que reduza o custo da instalação dos drenos e também do material usado, é altamente vantajosa. Diante disso, o objetivo desta pesquisa foi avaliar, em condições de laboratório, o desempenho hidráulico do sistema drenante constituído pelo tubo de PVC liso, próprio para esgoto doméstico, de $50,0 \mathrm{~mm}$ de diâmetro nominal e área aberta de $23,0 \mathrm{~cm}^{2} \mathrm{~m}^{-1}$, e por três tipos de envoltório. O desempenho do tubo drenante de PVC liso demonstrou que esse material é tecnicamente viável para drenagem agrícola, principalmente com o uso de brita como envoltório.
\end{abstract}

Palavras-chave: dreno, resistência de entrada, envelopes

\section{Smooth PVC tube with different envelopes as an alternative material in the subsurface drainage}

\begin{abstract}
In irrigated arid and semi-arid regions the drainage is, frequently, necessary to prevent the waterlogging of the soil and to control the salinity. One of the main obstacles in the installation of a subsurface drainage system is the high cost of the initial investment. Thus, some contribution that reduces the cost of the installation of the drains and also of the material used is highly advantageous. The objective of this research was to evaluate, under laboratory conditions, the hydraulic performance of the drainage system constituted by smooth PVC tube used for sewer with $50.0 \mathrm{~mm}$ of nominal diameter and open area of $23.0 \mathrm{~cm}^{2} \mathrm{~m}^{-1}$ and different types of envelopes, as an alternative material for subsurface drainage. The performance of the smooth PVC tube demonstrated that this material is technically viable for agricultural drainage principally when used with single stones as envelope.
\end{abstract}

Key words: drain, entrance resistance, envelope

\section{INTRODUÇÃO}

Em regiões áridas e semi-áridas, onde as precipitações são mal distribuídas no tempo e no espaço, torna-se necessário um planejamento agrícola eficiente, por meio do conhecimento das relações água-solo-planta-atmosfera, para otimizar o manejo dos recursos naturais, com destaque para o emprego adequado das técnicas de irrigação e drenagem.

Essas regiões são climaticamente caracterizadas por elevados níveis de evaporação que se sobrepõem aos de precipitação, sendo potencialmente propícias para os problemas da salinidade dos solos, uma vez que o perfil do solo não é naturalmente lavado o suficiente para promover a lixiviação dos sais solúveis da zona radicular efetiva das plantas cultivadas.

A ampliação das áreas irrigadas na região Nordeste do Brasil requer um manejo eficiente desta tecnologia para não tornar os solos salinos e/ou sódicos, mediante a utilização de águas de boa qualidade para a irrigação e aplicação de uma fração de lixiviação para o carreamento dos sais solúveis, os quais, a partir de certos níveis de concentração, são tóxicos para as culturas, o que pode ser obtido com a implantação de um sistema eficiente de drenagem subterrânea, uma vez que os solos são predominantemente estratificados e nem sempre apresentam uma drenagem natural adequada.

Um controle adequado da salinidade é de fundamental importância para o sucesso da agricultura irrigada e, para que 
assim seja, são necessárias práticas indispensáveis, como a aplicação de lâminas de água excedentes para lixiviação de sais, a utilização de culturas tolerantes à salinidade e a implantação de sistema de drenagem. A drenagem de terras agrícolas constitui, sem dúvida alguma, um complemento indispensável aos projetos de irrigação.

Manter uma produtividade satisfatória das culturas em áreas intensamente irrigadas, depende de uma drenagem adequada. A longo prazo, a sustentabilidade da atividade agrícola sem a drenagem é altamente questionável, em termos de se manter não só a integridade ambiental e o lençol freático baixo mas, também, a própria produtividade das culturas, devido aos riscos acelerados de encharcamento e salinidade na zona radicular efetiva das culturas (Manguerra \& Garcia, 1997). Esses dois riscos podem ser prevenidos por um controle melhor da água, assegurando-se que todo projeto de irrigação tenha uma drenagem adequada (Garcia et al., 1992; Datta et al., 2000).

Os três materiais predominantes para a confecção de tubos de drenagem de plástico são o policloreto de vinil (PVC), o polietileno de alta densidade (PEAD) e, em menor incidência, o polipropileno (PP). Em condições de altas temperaturas, os tubos de PEAD são mais afetados que os de PVC apresentando, conseqüentemente, risco maior de deformação. Por outro lado, o tubo de PVC se torna mais sensível a baixas temperaturas, tornando-se frágil quando exposto a temperaturas abaixo do ponto de congelamento da água (Dieleman \& Trafford, 1976; Cavelaars et al., 1994).

A utilização dos tubos de plástico lisos na drenagem iniciou-se em torno de 1960. Esses tubos foram depois substituídos por tubos de paredes corrugadas. A principal vantagem dos tubos plásticos sobre os de cerâmica ou de concreto, é que são mais leves, e isto faz com que o seu transporte e manuseio sejam mais fáceis, possibilitando altos ritmos de instalação.

A procura de técnicas e materiais capazes de reduzir os custos de implantação de sistemas de drenagem subterrânea, empregando-se drenos entubados e sem que haja perda da qualidade técnica do produto, deve constituir-se em preocupação constante dos técnicos envolvidos em trabalhos de drenagem, principalmente quando se tratar de drenagem de terras agrícolas. Assim, os tubos usados na drenagem agrícola podem ser, na maioria das vezes, substituídos por tubos de PVC rígidos e lisos, do tipo utilizado em esgoto doméstico, que são, geralmente, de custo inferior aos de seus concorrentes, além de apresentarem facilidade de manuseio e assentamento, o que resulta em redução nos custos de implantação. Um outro ponto positivo é a facilidade com que esse material pode ser encontrado no mercado (Batista et al., 1984).

Batista et al. (1984) ao estudarem, em condições de campo, o comportamento do tubo drenante constituído de PVC rígido de paredes lisas, de $50 \mathrm{~mm}$ de diâmetro próprio para esgoto, com envoltórios de brita $\mathrm{n}^{\circ} 1$ e de manta geotextil marca bidim OP-20 associada com a brita $\mathrm{n}^{\mathrm{o}} 1$, concluíram que o emprego desse tubo como material drenante, pode ser uma das soluções para a drenagem agrícola.

Assim, com o presente trabalho objetivou-se avaliar, sob condições de laboratório, as características hidráulicas do sistema drenante constituído por tubo de PVC liso próprio para esgoto doméstico de 50,0 mm de diâmetro nominal e 23,0 $\mathrm{cm}^{2} \mathrm{~m}^{-1}$ de área aberta e por três tipos de envoltório.

\section{MATERIAL E MÉTODOS}

Este trabalho foi realizado no Laboratório de Engenharia de Irrigação e Drenagem do Departamento de Engenharia Agrícola da Universidade Federal de Campina Grande (DEAg-CCTUFCG). Usou-se um modelo físico (Figura 1) composto de nove tanques construídos em alvenaria e internamente impermeabilizados com um produto líquido e viscoso, à base de tinta betuminosa. Cada tanque, medindo 0,8 $\mathrm{m}$ de comprimento, 1,0 $\mathrm{m}$ de largura e $0,9 \mathrm{~m}$ de altura, aplicável a uma condição de fluxo bidimensional, que é a mais usual de campo, foi construído baseado no modelo apresentado por Broughton et al. (1987).

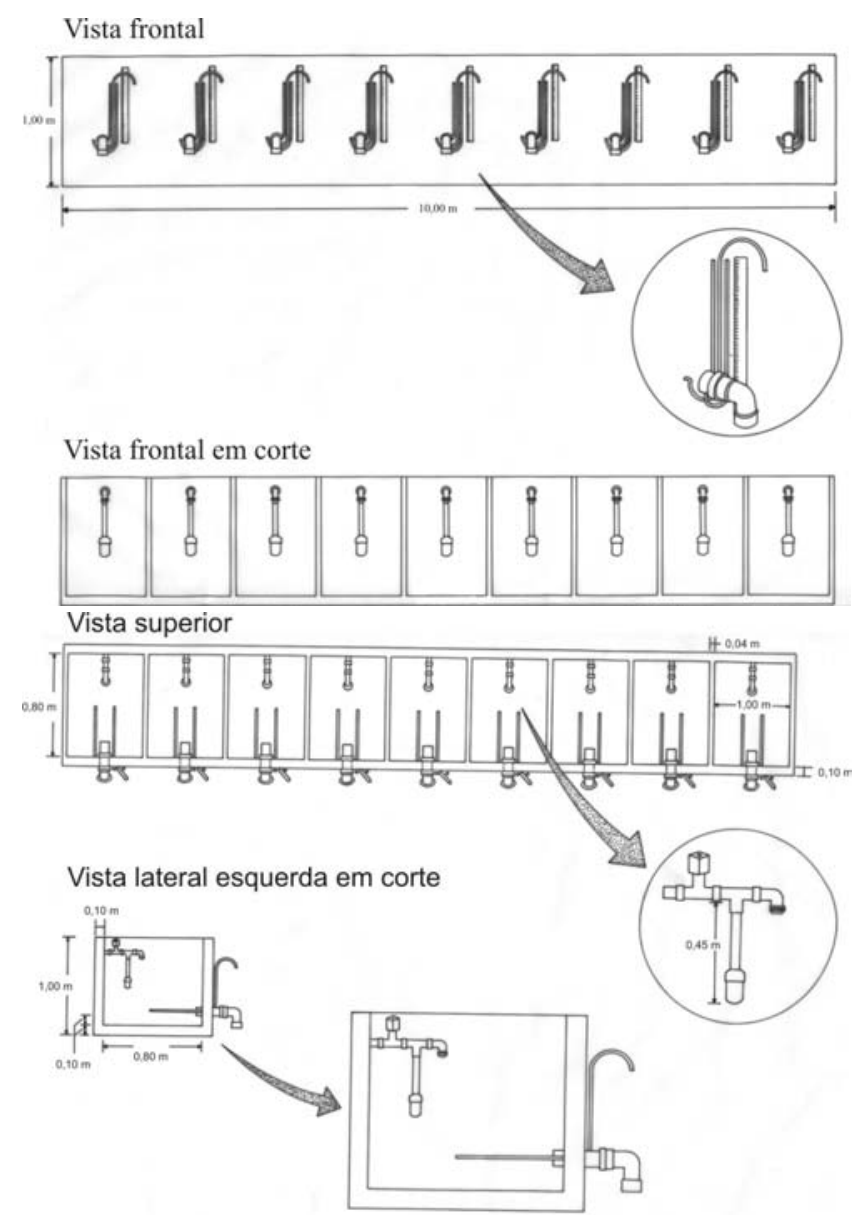

Figura 1. Ilustração do modelo físico

O meio poroso utilizado como material base nos ensaios, constituiu-se de areia secada ao ar, passada em uma peneira de malha com abertura de 2,0 mm, pesada e distribuída nos tanques, aleatoriamente, até a obtenção de $900 \mathrm{~kg}$, equivalente a um volume de $0,64 \mathrm{~m}^{3}$ por tanque e espessura de $0,8 \mathrm{~m}$. As características físico-hídricas do material poroso utilizado (Tabela 1) foram determinadas conforme metodologias adotadas pela Embrapa (1997).

Avaliaram-se três sistemas drenantes compostos por tubo de PVC liso, próprio para esgoto, de 50,0 $\mathrm{mm}$ de diâmetro 
Tabela 1. Características físico-hídricas do material poroso utilizado

\begin{tabular}{lr}
\hline Característica & Valor \\
\hline Areia grossa $\left(\mathrm{g} \mathrm{kg}^{-1}\right)$ & 210,00 \\
Areia fina $\left(\mathrm{g} \mathrm{kg}^{-1}\right)$ & 750,00 \\
Silte $\left(\mathrm{g} \mathrm{kg}^{-1}\right)$ & 20,00 \\
Argila $\left(\mathrm{g} \mathrm{kg}^{-1}\right)$ & 20,00 \\
Densidade global $\left(\mathrm{kg} \mathrm{dm}^{-3}\right)$ & 1,40 \\
Densidade real $\left(\mathrm{kg} \mathrm{dm}^{-3}\right)$ & 2,60 \\
Condutividade hidráulica $\left(\mathrm{m} \mathrm{d}^{-1}\right)$ & 26,78 \\
\hline
\end{tabular}

nominal e uma área aberta de $23,0 \mathrm{~cm}^{2} \mathrm{~m}^{-1}$ com nove fileiras de abertura, e de três tipos de envoltório. Cada sistema drenante, com comprimento de $0,6 \mathrm{~m}$, foi disposto horizontalmente, centralizado e nivelado a $10,0 \mathrm{~cm}$ do fundo do tanque, com três repetições. Utilizaram-se, como envoltórios, manta geotêxtil marca bidim OP-20, espuma e brita zero, sendo que os dois primeiros foram fixados nos tubos por meio de amarração manual, sob a forma de espiral, com linha de nylon de $0,5 \mathrm{~mm}$ de espessura. A espuma, com 5,0 mm de espessura utilizada nesta pesquisa como material envoltório alternativo, constituiuse de um material sintético à base de poliuretano, com diversas aplicações na indústria.

$\mathrm{Na}$ etapa em que se utilizou a brita zero como envoltório, foi necessário o uso de duas chapas de Duraplac com as dimensões de $0,76 \times 0,35 \times 0,03 \mathrm{~m}$, distanciadas $0,10 \mathrm{~m}$ a partir do tubo drenante, as quais serviram de gabarito na colocação desse envoltório. Nos tratamentos que receberam brita utilizou-se $0,050 \mathrm{~m}^{3}$ deste material, envolvendo todo o tubo drenante. Após atingir a espessura predeterminada, de $0,10 \mathrm{~m}$, o gabarito foi cuidadosamente retirado, complementando-se, assim, o volume do material poroso.

Por meio de brocas de 2,0 mm de diâmetro, utilizando-se um procedimento mecânico, em cada tubo drenante foram feitos 729 furos distribuídos em 9 filas, distanciados um do outro em aproximadamente $12,0 \mathrm{~mm}$ na longitudinal, de tal forma que uma unidade métrica de tubo dispusesse de $23,0 \mathrm{~cm}^{2}$ de área aberta total, valor este baseado no que dispõe a área aberta do tubo comercial de marca Drenoflex..

Para realização dos testes e se mantendo fechado o ponto de descarga do tanque, abriu-se o registro de alimentação até que o nível de encharcamento do material poroso fosse alcançado, o que era constatado pelo posicionamento dos níveis hidráulicos em piezômetros. Ao se atingir a condição de encharcamento, o fornecimento de água aos tanques era interrompido e o ponto de descarga aberto individualmente, de modo que houvesse o rebaixamento do nível freático e, conseqüentemente, o registro das leituras das cargas hidráulicas de entrada e total, e a coleta e pesagem do volume d'água em intervalos de tempo de descarga. Procedeu-se desta maneira, até que se verificasse uma descarga mínima em cada sistema drenante, correspondendo a um tempo de descarga de, em média, 1 h e 30 min por tanque.

Para cada sistema drenante instalado, foram avaliadas as cargas hidráulicas de entrada (he), a razão entre as cargas hidráulicas de entrada e total (he/htot), a resistência de entrada (re) e a descarga (q), para tempos acumulados que variaram de 39 a 1668 s. Para análise dessas variáveis utilizaram-se os critérios propostos por Wesseling \& van Someren (1972) e Dieleman \& Trafford (1976).
A carga hidráulica de entrada (he) foi avaliada mediante uma régua graduada instalada paralelamente aos piezômetros, localizada na parte externa do modelo físico. Tomou-se uma leitura direta do nível d'água no piezômetro inserido no sistema drenante. A componente resistência de entrada (re), por se constituir num parâmetro dos mais importantes como valor independente e como fração da carga hidráulica total, foi calculada mediante a equação abaixo, sugerida por Wesseling \& van Someren (1972):

$$
\text { re }=\frac{\text { he L }}{q}
$$

em que re é a resistência de entrada, em d $\mathrm{m}^{-1}$, he a carga hidráulica na entrada, em $\mathrm{m}$, q a descarga do dreno, em $\mathrm{m}^{3} \mathrm{~d}^{-1} \mathrm{e}$ L o comprimento do sistema drenante, em $\mathrm{m}$.

\section{RESULTADOS E DISCUSSÃO}

Os resultados da análise estatística referentes à carga hidráulica de entrada (he) e à descarga (q) apresentados na Tabela 2, mostram que houve diferença altamente significativa ( $1 \%$ de probabilidade) enquanto a razão entre as cargas hidráulicas de entrada e total (he/htot) e a resistência de entrada (re) apontam diferença significativa ( $5 \%$ de probabilidade) apenas entre os envoltórios avaliados, demonstrando que a carga hidráulica de entrada, a razão entre as cargas hidráulicas de entrada e total, a resistência de entrada e a descarga, dependem do tipo de envoltório utilizado. O coeficiente de variação da carga hidráulica de entrada (he) foi de 5,64\%, indicando uma ótima precisão experimental, segundo Ferreira (1991).

Tabela 2. Resumo da ANOVA das variáveis carga hidráulica de entrada (he), razão entre as cargas hidráulicas de entrada e total (he/htot), resistência de entrada (re) e descarga (q) do tubo de PVC liso

\begin{tabular}{lcccc}
\hline \multirow{2}{*}{ Causas de Variação } & \multicolumn{4}{c}{ Variáveis } \\
\cline { 2 - 5 } & he $(\mathrm{m})$ & he/htot & re $\left(\mathrm{dm}^{-1}\right)$ & $\mathrm{q}\left(\mathrm{m}^{3} \mathrm{~d}^{-1}\right)$ \\
\cline { 2 - 5 } & \multicolumn{4}{c}{ Teste F } \\
\hline Material envoltório & $34,81^{* *}$ & $6,95^{*}$ & $10,73^{*}$ & $272,84^{* *}$ \\
Q M do Resíduo & 0,00 & 0,00 & 0,00 & 0,82 \\
CV (\%) & 5,64 & 1,67 & 18,88 & 4,93 \\
\hline (*) Significan
\end{tabular}

Considerando-se que os envoltórios promoveram diferença significativa, compararam-se os valores médios das variáveis carga hidráulica de entrada (he), razão entre as cargas hidráulicas de entrada e total (he/htot), resistência de entrada (re) e descarga (q) mediante o teste de Tukey (Tabela 3). Nesta tabela, constata-se que o tubo de PVC liso apresentou comportamento similar com o uso dos envoltórios de bidim e de brita zero; já com o envoltório de espuma, a carga hidráulica de entrada (he) foi significativamente superior (28,81 e 43,96\% em relação aos envoltórios de bidim e brita zero, respectivamente) diferença esta que se deve, provavelmente, à maior resistência de entrada desse tipo de envoltório no tubo de PVC liso. 
Tabela 3. Valores médios da carga hidráulica de entrada (he), razão entre as cargas hidráulicas de entrada e total (he/htot), resistência de entrada (re) e descarga (q) do tubo de PVC liso, com diferentes envoltórios

\begin{tabular}{lrrr}
\hline \multirow{2}{*}{ Causas de Variação } & \multicolumn{3}{c}{ Envoltórios } \\
\cline { 2 - 4 } & \multicolumn{1}{c}{ Bidim } & Brita Zero & Espuma \\
\hline Carga hidráulica de entrada $(\mathrm{m})$ & $0,3193 \mathrm{~b}$ & $0,2857 \mathrm{~b}$ & $0,4113 \mathrm{a}$ \\
Razão he/htot & $0,8867 \mathrm{ab}$ & $0,8767 \mathrm{~b}$ & $0,9200 \mathrm{a}$ \\
Resistência de entrada $\left(\mathrm{d} \mathrm{m}^{-1}\right)$ & $0,0127 \mathrm{a}$ & $0,0068 \mathrm{~b}$ & $0,0146 \mathrm{a}$ \\
Descarga $\left(\mathrm{m}^{3} \mathrm{~d}^{-1}\right)$ & $12,0033 \mathrm{c}$ & $28,2567 \mathrm{a}$ & $14,9767 \mathrm{~b}$ \\
\hline Médias seguidas da mesma letra na linha não diferem a 5\% &
\end{tabular}

O menor valor da carga hidráulica de entrada foi obtido quando se utilizou o envoltório de brita zero, podendo ser explicado pela espessura de $10,0 \mathrm{~cm}$ desse envoltório envolvendo todo o tubo drenante, sua granulometria uniforme e poros relativamente grandes permitindo, assim, que ocorressem perdas reduzidas de carga na entrada do sistema drenante. De acordo com os critérios propostos por Dieleman \& Trafford (1976) o tubo de PVC liso apresentou desempenho regular com os envoltórios de brita e de manta geotêxtil marca bidim, porque a carga hidráulica de entrada variou entre $0,15 \mathrm{e}$ $0,30 \mathrm{~m}$. Ressalta-se que, para a carga hidráulica de entrada, os envoltórios de brita e de manta geotêxtil marca bidim não diferiram a $5 \%$ de probabilidade; já para o envoltório de espuma, esse tubo apresentou desempenho ruim, uma vez que a carga hidráulica de entrada foi $0,4113 \mathrm{~m}$. Esses resultados concordam com os obtidos por Gonzaga (1994).

A análise da variância da razão entre as cargas hidráulicas de entrada e total (he/htot) mostrada na Tabela 2, revela que os envoltórios apresentaram diferença significativa. $\mathrm{O}$ coeficiente de variação foi de 1,67\%, indicando ótima precisão experimental, segundo Ferreira (1991). Constatou-se que os valores médios dessa razão foram próximos da unidade, tendo em vista os piezômetros adjacentes ao sistema drenante se encontrarem dispostos bem próximos. Adotando-se os critérios propostos por Dieleman \& Trafford (1976) para se avaliar a razão entre as cargas hidráulicas de entrada e total (he/htot) observa-se que os resultados não foram satisfatórios, porque he/htot foi superior a 0,60 para os três tipos de envoltório estudados. $O$ resultado dessa avaliação é explicado pelo fato da condição desses critérios diferir daquela em que foram submetidos os sistemas drenantes neste estudo, qual seja, um meio poroso (areia) com elevada condutividade hidráulica $\left(26,78 \mathrm{~m} \mathrm{~d}^{-1}\right)$ e um espaçamento entre drenos de $1,0 \mathrm{~m}$. Esses resultados, no entanto, concordam com os obtidos por Gonzaga (1994) e Silva (1997).

A Figura 2 apresenta a relação entre a resistência de entrada e a carga hidráulica total do tubo de PVC liso, com diferentes envoltórios, e nela se percebe comportamento diferenciado desse tubo drenante, com todos os materiais envoltórios. A menor e a maior resistência de entrada foram obtidas quando se utilizaram os envoltórios de brita zero e espuma, respectivamente. A menor resistência oferecida com o uso da brita zero pode ser explicada pelo fato desse tipo de envoltório ter apresentado um raio efetivo maior, granulometria uniforme e poros relativamente grandes, enquanto a maior resistência observada no envoltório de espuma, quando comparada com a brita zero e a manta geotexxtil (bidim) se explica, provavelmente, por não ter existido uma aderência perfeita desse envoltório no tubo drenante, embora não tenha havido diferença estatística entre os envoltórios de manta geotêxtil (bidim) e espuma (Tabela 3), fato que demonstra que, do ponto de vista da resistência de entrada, é indiferente o uso da manta geotêxtil (bidim) ou da espuma no tubo drenante de PVC liso, uma vez que esses envoltórios apresentaram resposta similar. Como a resistência de entrada é considerada um dos mais importantes parâmetros de avaliação de sistemas drenantes, conclui-se que o tubo drenante de PVC liso com a utilização desses tipos de envoltório apresenta características desejáveis como material drenante, porque os valores de resistência de entrada encontrados são considerados bons ( $\mathrm{re}<0,75 \mathrm{~d} \mathrm{~m}^{-1}$ ) e muito bons (re $<0,475 \mathrm{~d} \mathrm{~m}^{-1}$ ), respectivamente, conforme os critérios propostos por Dieleman \& Trafford (1976) e Wesseling \& van Someren (1972).

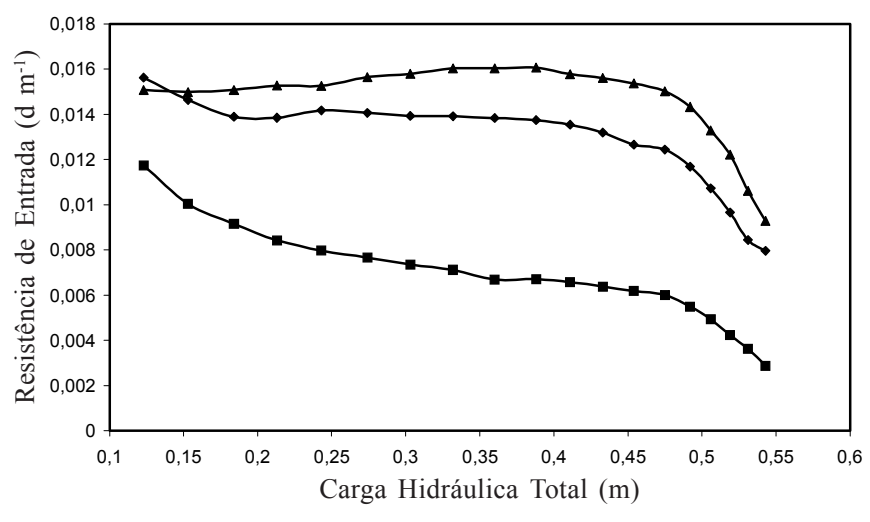

$\rightarrow$-Bidim $\rightarrow$-Brita zero $\leftarrow$-Espuma

Figura 2. Curvas da resistência de entrada $\left(\mathrm{d} \mathrm{m}^{-1}\right)$ em relação à carga hidráulica total (m) do tubo de PVC liso, com diferentes envoltórios

Tem-se, na Figura 3, as curvas da descarga em função do tempo acumulado de drenagem do tubo de PVC liso, com os envoltórios de manta geotêxtil (bidim), brita zero e espuma, observando-se que a descarga teve comportamento característico, inicialmente com altas taxas e, em seguida, decrescendo gradualmente, até alcançar um valor praticamente constante. Percebe-se, na Figura 3, que a descarga alcançou um valor máximo em torno de $100,0 \mathrm{~m}^{3} \mathrm{~d}^{-1}$ quando a brita zero foi utilizada como envoltório, evidenciando uma condição altamente desejável, em termos de rebaixamento do lençol freático o que, por sua vez, indica que a brita zero, como envoltório, faz com que o sistema de drenagem se torne efetivo ao remover o excesso de água do perfil do solo em um tempo relativamente rápido; quando, porém, se fez uso dos envoltórios de manta geotêxtil (bidim) e de espuma, seus valores máximos obtidos foram, respectivamente, 27,0 e $35,0 \mathrm{~m}^{3} \mathrm{~d}^{-1}$ demonstrando, portanto, um rebaixamento maior do lençol freático com o uso da espuma, quando comparado com a manta geotêxtil (bidim) revelando-se a espuma como material possivelmente promissor como envoltório para a drenagem agrícola. A Tabela 3 apresenta os valores médios da descarga obtidos pelo teste de Tukey, na qual se observa que o tubo de PVC liso com o envoltório de bidim, apresentou a menor descarga entre os envoltórios avaliados. 


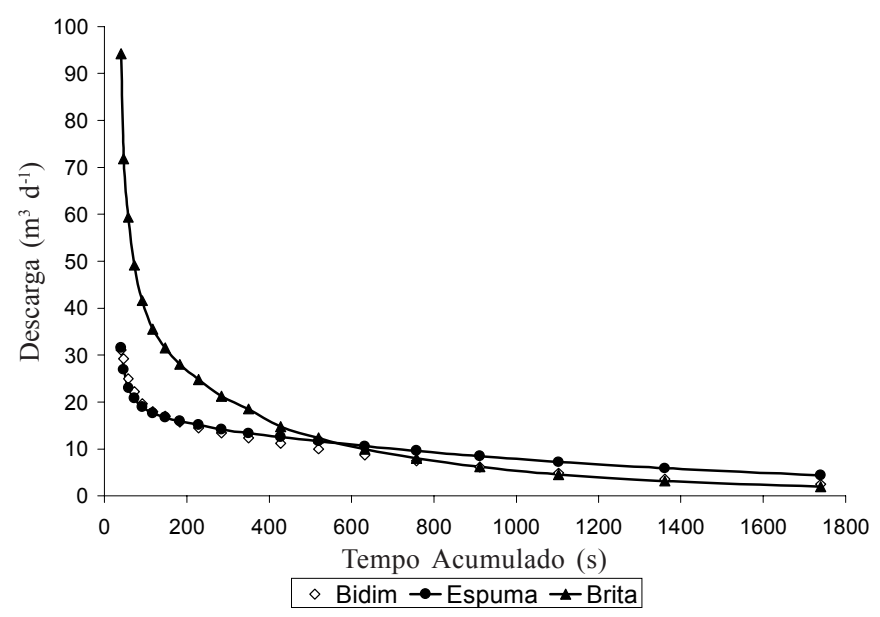

Figura 3. Curvas da descarga $\left(\mathrm{m}^{3} \mathrm{~d}^{-1}\right)$ em relação ao tempo acumulado de drenagem (s) do tubo de PVC liso, com diferentes envoltórios

Em condições de laboratório, o tubo de PVC liso, próprio para esgoto doméstico, revelou-se tecnicamente com elevado grau de potencialidade para a drenagem agrícola; acrescentase, ainda, o fato de ser um material de fácil obtenção no mercado local, além de seu preço ser bastante inferior quando comparado com os materiais drenantes convencionais, podendo ser adquirido diretamente do fabricante e reduzindo, então, o custo do material. Assim sendo, o custo para implantação de um projeto de drenagem agrícola será menor, tendo em vista o preço da tubulação não se constituir em um obstáculo para efetivação da prática da drenagem.

\section{CONCLUSÕES}

Os resultados obtidos permitem as seguintes conclusões a respeito do tubo de PVC liso, próprio para esgoto doméstico, como material drenante:

1. Mostrou-se viável como material alternativo para a drenagem agrícola, em condições de laboratório.

2. Apresentou carga hidráulica de entrada considerada regular, para todos os envoltórios utilizados.

3. Apresentou resistência de entrada classificada entre boa a muito boa.

4. Apresentou uma descarga maior e uma menor, com o uso dos envoltórios de brita zero e de manta geotêxtil (bidim) respectivamente.

5. Apresentou resistência de entrada classificada entre boa a muito boa, para os três tipos de envoltório estudados; entretanto, para o envoltório de espuma a resistência de entrada foi superior em relação aos demais envoltórios.

\section{LITERATURA CITADA}

Batista, M. de J.; Silva, C.L. da; Rodrigues, J.D.I. Análise técnico-econômica do comportamento de drenos entubados empregando envelope somente de cascalho e de cascalho com manta sintética. Irrigação e Tecnologia Moderna, Brasília, n.19, p.14-22, 1984.

Broughton, R.S.; Chirada, K.E; Bonnell, R.B. Test of drain tubes with pin holes and small slots. In: Drainage design and manegement. St. Joseph. ASAE. Publication 7, 1987. p.362371.

Cavelaars, J.C.; Vlotman, W.F. ; Spoor, G. Subsurface drainage systems. In: Ritzema, H.P. (ed.). Drainage principles and applications. Wageningen: ILRI 1994. p.827-929. Publication 16

Datta, K.K.; Jong, C. de; Singh, O.P. Reclaiming salt-affected land through drainage in Haryana, India: a financial analysis. Agricultural Water Management, Amesterdam, v.46, n.1, p.55-71, 2000.

Dieleman, P.J.; Trafford, B.D. Ensayos de drenaje. Rome: FAO, 1976. 172p. Irrigation and Drainage, Paper 28

EMBRAPA - Empresa Brasileira de Pesquisa Agropecuária Serviço Nacional de Levantamento e Conservação de Solos. Manual de métodos de análise de solos. Rio de Janeiro, 1997.212p.

Ferreira, P.V. Estatística experimental aplicada à agronomia. Maceió: EDUFAL, 1991.437p.

Garcia, L.A.; Gates, T.K.; Jensen, M.E.; Podmore, T.H. Management support for conjunctive irrigation and drainage. In: Drainage and water table control. Proceedings of the International Drainage Symposium, 6. ASAE. St. Joseph, 1992. p.12-19.

Gonzaga, E. Aplicabilidade de tubo de PVC para esgoto, como material drenante alternativo. Campina Grande: UFPB, 1994, 60p. Dissertação Mestrado

Manguerra, H.B.; Garcia, L.A. Field strategy for agricultural drainage and water-quality management. Journal of Irrigation and Drainage Engineering of ASCE, New York, v.123, n.1, p.37-44, 1997.

Silva, V.de P. Avaliação do desempenho de sistemas drenantes em condições de laboratório. Campina Grande: UFCG, 1997, 121p. Dissertação Mestrado

Wesseling, J.; van Someren, C.L. Drainage materials. Rome: FAO, 1972. p.55-83. Irrigation and Drainage, Paper 9 